\title{
Rotational Constraint between Beams in 3-D Space
}

\author{
Hamid Reza Motamedian and Artem Kulachenko \\ Department of Solid Mechanics, Royal Institute of Technology (KTH), 10044 Stockholm, Sweden \\ Correspondence: Hamid Reza Motamedian (hrmo@kth.se)
}

Received: 8 August 2017 - Revised: 30 April 2018 - Accepted: 10 October 2018 - Published: 16 November 2018

\begin{abstract}
In this paper, we develop two alternative formulations for the rotational constraint between the tangents to connected beams with large deformations in 3-D space. Such a formulation is useful for modeling bonded/welded connections between beams. The first formulation is derived by consistently linearizing the variation of the strain energy and by assuming linear shape functions for the beam elements. This formulation can be used with both the Lagrange multiplier and the penalty stiffness method. The second non-consistent formulation assumes that the contact normal is independent of the nodal displacements at each iteration, and is updated consistently between iterations. In other words, we ignore the contribution due to the change of the contact normal in the linearization of the contact gap function. This assumption yields simpler equations and requires no specific assumption regarding the shape functions for the underlying beam elements. However, it is limited to the penalty method. We demonstrate the performance of the presented formulations in solving problems using implicit time integration. We also present a case showing the implications of ignoring this rotational constraint in modeling a network of beams.
\end{abstract}

\section{Introduction}

The beam-to-beam contact formulation was originally discussed by Wriggers and Zavarise (1997) for the case of normal contact between 3-D beams. This formulation was later extended by Zavarise and Wriggers (2000) to include the tangential contact of linear beams in 3-D space. These formulations assumed circular cross-sections for the beams, but they were developed further by Litewka and Wriggers (2002) to analyze beams with rectangular cross-sections. Subsequently, Litewka used the formulations with the Lagrange and penalty stiffness methods (Litewka, 2005) and used smoothing procedures to solve the divergence problem that can occur when the contact point moves from one element to another (Litewka, 2006, 2007, 2010). Also self contacting was formulated for beams experiencing loop formation by Gay Neto et al. (2015) as an extension of the previously presented formulations. Recently Motamedian (2018) suggested a robust algorithm with a penalty method and the assumption that the contact point, normal, and tangent vectors are constant (independent of deformations) at each iteration. This assumption made it possible to derive an easy-toimplement formulation for the normal and tangential contact of beams suitable for any appropriate shape function.
In all the above mentioned papers, a pointwise contact between the fibers was assumed without taking into account the moment transfer at the point of contact. Although the effect of a rotational contact might be negligible in some cases, it is an important factor for a number of applications, e.g. for modeling welded connections between beams or for considering joints in a fiber network (Magnusson and Östlund, 2013).

The mentioned beam-to-beam contact formulations are generally search for a contact point based on the shortest distance between elements. In case of parallel or almost parallel beams, these methods will not work, as a unique contact point cannot be found. To handle this problem, instead of point-to-point methods, point-to-line and line-to-line algorithms are used. For example Durville suggested defining an intermediate geometry between the contacting beams and using a master/slave type of procedure between each beam and this intermediate geometry (Durville, 2012, 2010). This method treats the beams symmetrically and is useful when beams are locally almost parallel at the contact location. Meier and Popp (2016) found a criteria based on the angle between beams, their curvature and radius of cross-section to determine if a point-to-point formulation is applicable to 
a pair of beams in contact. They also suggested a line-to-line procedure for the contact of beams (Meier and Popp, 2016). This line-to-line procedure can handle small contact angles well, however it loses efficiency in comparison with point-topoint algorithms when the contact angle increases. To benefit from the advantages of both algorithms, Meier et al. (2017) suggested a method to combine both formulations into one.

Konyukhov and Schweizerhof (2010) presented a geometrically exact covariant approach for edge-to-edge contact which can include rotational constraints. The formulation requires the closest point projection procedure in the local Serret-Frenet coordinate system attached to the curves, in order to derive measures of contact interactions. The consistent application of this approach requires use of high-order finite element techniques to ensure $\mathrm{C} 1$ continuity.

Although the term contact usually refers to an inequality constraint, when inseparable/adhesive connections between bodies are considered, an equality constraint has to be satisfied. This case is especially applicable when studying beams connected in complex assemblies or for modeling multiple fiber-to-fiber contacts in simulating wires or fiber-based composite materials.

In this paper, we present the formulation for a constraint between the tangents to the beams at their point of connection. This constraint keeps the angle between the tangents constant, or in other words, avoids relative rotation of the tangents. We use the term rotational constraint in the rest of this article to refer to this equality constraint. This rotational constraint formulation can easily be expanded into a rotational contact formulation by considering a criterion which can activate or deactivate the contributions from this constraint during the system evolution.

Consequently, we first present a weak formulation for both the Lagrange multiplier and the penalty stiffness method. Lagrange multiplier and penalty stiffness are two standard methods, successfully used with FEM for contact analyses. Next, we suggest two alternative formulations for the rotational constraint between beams. In the first method, based on the assumption of linear shape functions for the beams, a rotational gap is defined, and using the variational methods and by linearizing the nonlinear equations, the consistent tangent stiffness matrix is derived for both the Lagrange multiplier and the penalty stiffness methods. In the second method a non-consistent approach is used. In this method the contact normal is updated consistently between successive iterations, however the contributions from the change of the contact normal are ignored when linearizing the gap function. In other words, we assume that the contact normal is independent of the deformation at each iteration. We present this simpler formulation with the penalty stiffness method. We show the efficiency of both formulations by solving several examples and demonstrate the effect of including the rotational constraint in the simulation of a network of randomly oriented, interconnected fibers.

\section{The Weak Formulation}

The weak formulation of the rotational constraint is presented in this section and will be used in the derivation of the tangent stiffness matrices, using both presented formulations, in the following section.

The total potential energy, $\Pi$, is the sum of the elastic strain energy, $U$, stored in the deformed body and the potential of the external forces, $V$, associated with the applied forces:

$\Pi=U+V$.

The principle of stationary potential energy states that an infinitesimal variation from the equilibrium position involves no change in the energy:

$\delta \Pi=\delta U+\delta V=0$.

In general, Eq. (2) is nonlinear and an iterational scheme like Newton-Raphson is used to solve this equation:

$\delta \Pi^{(i)}=\delta \Pi^{(i-1)}+\Delta \delta \Pi^{(i)}=0$,

where $(i-1)$ and $(i)$ refer to iteration numbers. Two of the methods mainly used to solve such problems are penalty stiffness and Lagrange multiplier methods as described below.

\subsection{Penalty Stiffness Method}

In penalty stiffness method, it is assumed that a high-stiffness spring is connected between the contacting points. In this case, the elastic energy can be written as:

$U=U_{\mathrm{b} 1}\left(\boldsymbol{u}_{1}\right)+U_{\mathrm{b} 2}\left(\boldsymbol{u}_{2}\right)+U_{\mathrm{c}}$,

where $U_{\mathrm{b} 1}\left(\boldsymbol{u}_{1}\right)$ and $U_{\mathrm{b} 2}\left(\boldsymbol{u}_{2}\right)$ are the strain energy of the first and the second beam respectively and $U_{\mathrm{c}}$ is the strain energy due to contact (stored in the spring), which can be split into normal, tangential, rotational (constraint between tangents to the beams), as well as other contributions (such as out-ofplane or torsional rotations which are ignored here):

$U_{\mathrm{c}}=U_{\mathrm{Nc}}+U_{\mathrm{Tc}}+U_{\mathrm{Rc}}+\ldots$,

where the strain energy due to normal contact, $U_{\mathrm{Nc}}$, and tangential contact, $U_{\mathrm{Tc}}$, have already been discussed in Wriggers and Zavarise (1997), Zavarise and Wriggers (2000), Motamedian (2018) for beams with circular cross-sections. As a result, to formulate the finite element equations, we just need to find $\delta U_{\mathrm{Rc}}$ and $\Delta \delta U_{\mathrm{Rc}}$ in terms of the nodal displacements. We have the following equation for $U_{\mathrm{Rc}}$ :

$U_{\mathrm{Rc}}=\frac{1}{2} \varepsilon_{\mathrm{R}} g_{\mathrm{R}}^{2}$

where $g_{\mathrm{R}}$ is the rotational gap and $\varepsilon_{\mathrm{R}}$ is the rotational penalty stiffness. This equation leads to the following contributions of the rotational constraint:

$\delta U_{\mathrm{Rc}}=\varepsilon_{\mathrm{R}} g_{\mathrm{R}} \delta g_{\mathrm{R}}$

$\Delta \delta U_{\mathrm{Rc}}=\varepsilon_{\mathrm{R}} \Delta g_{\mathrm{R}} \delta g_{\mathrm{R}}+\varepsilon_{\mathrm{R}} g_{\mathrm{R}} \Delta \delta g_{\mathrm{R}}$ 


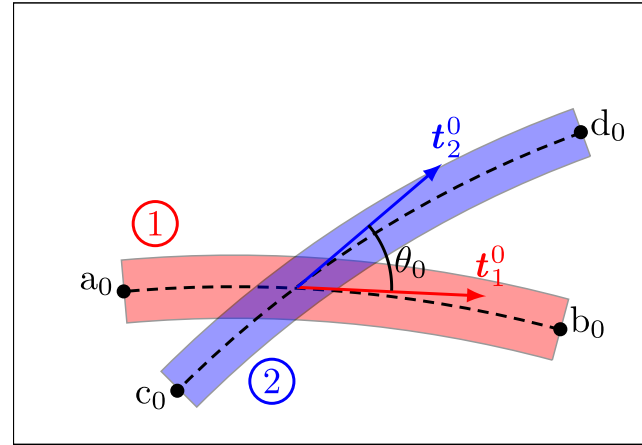

(a)

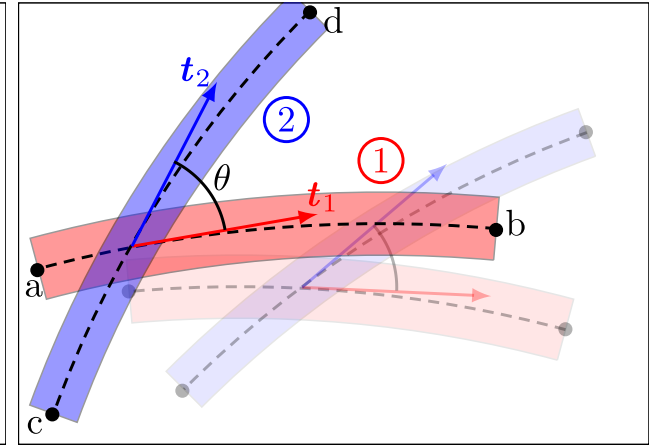

(b)

Figure 1. Definition of parameters in (a) the initial and (b) the current configurations of beams in contact. The beams are viewed along the contact normal.

\subsection{Lagrange Multiplier Method}

We want to solve the Eq. (2) subjected to the constraint $g_{\mathrm{R}}=$ 0 . Using Lagrange multiplier method, we can enforce this constraint by solving the following equation instead:

$\delta\left(\Pi+\lambda_{\mathrm{R}} g_{\mathrm{R}}\right)=\delta \Pi+\delta\left(\lambda_{\mathrm{R}} g_{\mathrm{R}}\right)=0$,

where $\lambda_{R}$ is the lagrange multiplier. The second term in this equation can be calculated as:

$\delta\left(\lambda_{\mathrm{R}} g_{\mathrm{R}}\right)=\delta \lambda_{\mathrm{R}} g_{\mathrm{R}}+\lambda_{\mathrm{R}} \delta g_{\mathrm{R}}$

To solve Eq. (9) using Newton-Raphson method, $\Delta \delta\left(\lambda_{\mathrm{R}} g_{\mathrm{R}}\right)$ needs to be calculated as follows:

$\Delta \delta\left(\lambda_{\mathrm{R}} g_{\mathrm{R}}\right)=\delta \lambda_{\mathrm{R}} \Delta g_{\mathrm{R}}+\Delta \lambda_{\mathrm{R}} \delta g_{\mathrm{R}}+\lambda_{\mathrm{R}} \Delta \delta g_{\mathrm{R}}$

Note that while with the first (consistent approach) formulation presented in Sect. (3), both the Lagrange multiplier and penalty stiffness methods can be used, the second formulation (non-consistent approach) is derived only for the penalty stiffness method. Using either the penalty stiffness or Lagrange multiplier method, we need to find $g_{\mathrm{R}}, \delta g_{\mathrm{R}}, \Delta g_{\mathrm{R}}$, and $\Delta \delta g_{\mathrm{R}}$ in terms of the nodal displacements of the beam elements in contact.

\section{The Rotational Tangent Stiffness Matrix}

We can define the rotational gap function, $g_{\mathrm{R}}$, as the change in the angle between the tangents to the beams at the contact point:

$g_{\mathrm{R}}=\theta-\theta_{0}$,

where $\theta_{0}$ and $\theta$ are the initial and current angles between the tangents to the beams at the contact point, as shown in Fig. 1. We will use two different approaches to derive the tangent stiffness matrix for the rotational constraint. These two approaches have the following assumptions in common:
- The contact between the beam elements is pointwise.

- No pair of beam elements can be in contact at more than one point. (If two beam elements have more that one contact point, we need to divide at least one of these elements into two or more elements, to ensure no more than one single contact point between each pair of elements. Note that it is possible for an element to have several contact points with different elements.)

Please note that both the consistent and non-consistent derivations in the following sections are independent of the beam formulation and all the parameter (nodal coordinates, displacements, vectors, ...) are assumed to be in the global coordinate system.

\subsection{Method 1: Consistent Approach}

In this approach, in addition to the assumptions mentioned above, we assume that the beam elements have linear shape functions, both for their geometry and displacements (this is the same assumption as in Zavarise and Wriggers, 2000), to simplify the derivation. As a result, the unit tangent vectors to the beams (at the contact point) can be written as follows:

$\left\{\begin{array}{l}t_{1}=\frac{x_{\mathrm{b}}-x_{\mathrm{a}}}{\left\|\left(x_{\mathrm{b}}-x_{\mathrm{a}}\right)\right\|}=\frac{x_{\mathrm{b}}-x_{\mathrm{a}}}{l_{1}} \\ t_{2}=\frac{x_{\mathrm{d}}-x_{\mathrm{c}}}{\left\|\left(x_{\mathrm{d}}-x_{\mathrm{c}}\right)\right\|}=\frac{x_{\mathrm{d}}-x_{\mathrm{c}}}{l_{2}}\end{array}\right.$

where, as shown in Fig. $1, t_{1}$ and $t_{2}$ are the tangents to Beams 1 and 2, $\boldsymbol{x}_{\mathrm{a}}$ and $\boldsymbol{x}_{\mathrm{b}}$ are the current positions of the starting and end nodes of Beam 1, $\boldsymbol{x}_{\mathrm{c}}$ and $\boldsymbol{x}_{\mathrm{d}}$ are the current positions of the starting and end nodes of Beam 2 , and $l_{1}$ and $l_{2}$ are the current lengths of Beams 1 and 2, respectively. To find the variation of the rotational gap, $\delta g_{R}$, (similarly for $\left.\Delta g_{\mathrm{R}}\right)$, we have

$\delta g_{\mathrm{R}}=\delta\left(\theta-\theta_{0}\right)=\delta \theta-\delta \theta_{0}=\delta \theta-0=\delta \theta$ 
We know that the inner product of two unit vectors gives the cosine of the angle between them:

$\cos \theta=t_{1} \cdot t_{2}$

Using this equation, we can find the variation of the angle between the two tangent vectors as follows:

$$
\begin{aligned}
& \left\{\begin{array}{l}
\delta(\cos \theta)=\delta\left(\boldsymbol{t}_{1} \cdot \boldsymbol{t}_{2}\right)=\delta \boldsymbol{t}_{1} \cdot \boldsymbol{t}_{2}+\boldsymbol{t}_{1} \cdot \delta \boldsymbol{t}_{2} \\
\delta(\cos \theta)=-\sin \theta \delta \theta
\end{array}\right. \\
& \Rightarrow \delta \theta=\delta g_{\mathrm{R}}=-\frac{\delta \boldsymbol{t}_{1} \cdot \boldsymbol{t}_{2}+\boldsymbol{t}_{1} \cdot \delta \boldsymbol{t}_{2}}{\sin \theta}=-\frac{\delta \boldsymbol{t}_{1} \cdot \boldsymbol{t}_{2}+\boldsymbol{t}_{1} \cdot \delta \boldsymbol{t}_{2}}{\sqrt{1-\left(\boldsymbol{t}_{1} \cdot \boldsymbol{t}_{2}\right)^{2}}}
\end{aligned}
$$

As shown in Appendix A1, the variation of the unit tangent vectors $t_{1}$ and $t_{2}$ can be calculated by

$$
\left\{\begin{array}{l}
\delta \boldsymbol{t}_{1}=\frac{1}{l_{1}}\left(\mathbf{I}-\boldsymbol{t}_{1} \otimes \boldsymbol{t}_{1}\right) \cdot\left(\delta \boldsymbol{u}_{\mathrm{b}}-\delta \boldsymbol{u}_{\mathrm{a}}\right) \\
\delta \boldsymbol{t}_{2}=\frac{1}{l_{2}}\left(\mathbf{I}-\boldsymbol{t}_{2} \otimes \boldsymbol{t}_{2}\right) \cdot\left(\delta \boldsymbol{u}_{\mathrm{d}}-\delta \boldsymbol{u}_{\mathrm{c}}\right)
\end{array}\right.
$$

Substituting Eqs. (17) into (16), we can find $\delta g_{\mathrm{R}}$ :

$$
\begin{aligned}
& \delta g_{\mathrm{R}}=\frac{-1}{\sqrt{1-\alpha^{2}}} \\
& \quad\left(\frac{1}{l_{1}}\left(\boldsymbol{t}_{2}-\alpha \boldsymbol{t}_{1}\right) \cdot\left(\delta \boldsymbol{u}_{\mathrm{b}}-\delta \boldsymbol{u}_{\mathrm{a}}\right)+\frac{1}{l_{2}}\left(\boldsymbol{t}_{1}-\alpha \boldsymbol{t}_{2}\right) \cdot\left(\delta \boldsymbol{u}_{\mathrm{d}}-\delta \boldsymbol{u}_{\mathrm{c}}\right)\right)
\end{aligned}
$$

where the notation $\alpha=\cos \theta=\boldsymbol{t}_{1} \cdot \boldsymbol{t}_{2}$ is used to shorten the equation. Using matrix notation and defining $\boldsymbol{u}=\left[\begin{array}{l}\boldsymbol{u}_{1} \\ \boldsymbol{u}_{2}\end{array}\right]$ with $\boldsymbol{u}_{1}=\left[\begin{array}{l}\boldsymbol{u}_{\mathrm{a}} \\ \boldsymbol{u}_{\mathrm{b}}\end{array}\right]$ and $\boldsymbol{u}_{2}=\left[\begin{array}{l}\boldsymbol{u}_{\mathrm{c}} \\ \boldsymbol{u}_{\mathrm{d}}\end{array}\right]$, we can rewrite Eq. (18):

$\delta g_{\mathrm{R}}=\frac{1}{\sqrt{1-\alpha^{2}}} \delta \boldsymbol{u}^{\mathrm{T}}\left[\begin{array}{r}\frac{1}{l_{1}}\left(\boldsymbol{t}_{2}-\alpha \boldsymbol{t}_{1}\right) \\ -\frac{1}{l_{1}}\left(\boldsymbol{t}_{2}-\alpha \boldsymbol{t}_{1}\right) \\ \frac{1}{l_{2}}\left(\boldsymbol{t}_{1}-\alpha \boldsymbol{t}_{2}\right) \\ -\frac{1}{l_{2}}\left(\boldsymbol{t}_{1}-\alpha \boldsymbol{t}_{2}\right)\end{array}\right]=\delta \boldsymbol{u}^{\mathrm{T}} \mathbf{G}^{\mathrm{T}}$

Similarly, $\Delta g_{\mathrm{R}}$ can be written as

$\Delta g_{\mathrm{R}}=\mathbf{G} \Delta \boldsymbol{u}$

To find $\Delta \delta g_{\mathrm{R}}$, we use Eq. (16) and the following change of variables:

$$
\begin{aligned}
& \delta g_{\mathrm{R}}=-\frac{A}{B} \\
& A=\delta \boldsymbol{t}_{1} \cdot \boldsymbol{t}_{2}+\boldsymbol{t}_{1} \cdot \delta \boldsymbol{t}_{2} \\
& B=\sqrt{1-\left(\boldsymbol{t}_{1} \cdot \boldsymbol{t}_{2}\right)^{2}}
\end{aligned}
$$

As a result, we can write $\Delta \delta g_{\mathrm{R}}$ as

$$
\Delta \delta g_{\mathrm{R}}=-\frac{(\Delta A) B-A(\Delta B)}{B^{2}}=-\frac{1}{B}\left(\Delta A+\delta g_{\mathrm{R}} \Delta B\right)
$$

Now we need to calculate $\Delta A$ and $\Delta B$.

$$
\Delta A=\Delta \delta t_{1} \cdot t_{2}+\delta t_{1} \cdot \Delta t_{2}+\Delta \delta t_{2} \cdot t_{1}+\delta t_{2} \cdot \Delta t_{1}
$$

Using the results obtained in Appendices A3, A4, and the notation $\boldsymbol{t}_{i j}=\boldsymbol{t}_{i} \otimes \boldsymbol{t}_{j}(i, j=1,2)$ to shorten the equation, we can write $\Delta A$ as

$$
\begin{aligned}
\Delta A= & \frac{-1}{L_{1}^{2}}\left(\delta \boldsymbol{u}_{\mathrm{b}}-\delta \boldsymbol{u}_{\mathrm{a}}\right) \cdot\left(\alpha \mathbf{I}+\boldsymbol{t}_{12}-2 \alpha \boldsymbol{t}_{11}\right) \\
& \cdot\left(\Delta \boldsymbol{u}_{\mathrm{b}}-\Delta \boldsymbol{u}_{\mathrm{a}}\right) \\
+ & \frac{1}{L_{1} L_{2}}\left(\delta \boldsymbol{u}_{\mathrm{b}}-\delta \boldsymbol{u}_{\mathrm{a}}\right) \cdot\left(\mathbf{I}-\boldsymbol{t}_{11}-\boldsymbol{t}_{22}+\alpha \boldsymbol{t}_{12}\right) \\
& \cdot\left(\Delta \boldsymbol{u}_{\mathrm{d}}-\Delta \boldsymbol{u}_{\mathrm{c}}\right) \\
+ & \frac{-1}{L_{2}^{2}}\left(\delta \boldsymbol{u}_{\mathrm{d}}-\delta \boldsymbol{u}_{\mathrm{c}}\right) \cdot\left(\alpha \mathbf{I}+\boldsymbol{t}_{21}-2 \alpha \boldsymbol{t}_{22}\right) \cdot\left(\Delta \boldsymbol{u}_{d}-\Delta \boldsymbol{u}_{c}\right) \\
+ & \frac{1}{L_{1} L_{2}}\left(\delta \boldsymbol{u}_{\mathrm{d}}-\delta \boldsymbol{u}_{\mathrm{c}}\right) \cdot\left(\mathbf{I}-\boldsymbol{t}_{11}-\boldsymbol{t}_{22}+\alpha \boldsymbol{t}_{21}\right) \\
& \cdot\left(\Delta \boldsymbol{u}_{\mathrm{b}}-\Delta \boldsymbol{u}_{\mathrm{a}}\right)
\end{aligned}
$$

Written in matrix notation, we have the following equation:

$$
\begin{aligned}
\Delta A & =\delta \boldsymbol{u}^{\mathrm{T}}\left[\begin{array}{rrrr}
\mathbf{P}_{1} & -\mathbf{P}_{1} & \mathbf{P}_{3} & -\mathbf{P}_{3} \\
-\mathbf{P}_{1} & \mathbf{P}_{1} & -\mathbf{P}_{3} & \mathbf{P}_{3} \\
\mathbf{P}_{4} & -\mathbf{P}_{4} & \mathbf{P}_{2} & -\mathbf{P}_{2} \\
-\mathbf{P}_{4} & \mathbf{P}_{4} & -\mathbf{P}_{2} & \mathbf{P}_{2}
\end{array}\right] \Delta \boldsymbol{u} \\
& =\delta \boldsymbol{u}^{\mathrm{T}} \mathbf{Q}_{1} \Delta \boldsymbol{u}
\end{aligned}
$$

where

$$
\begin{aligned}
& \mathbf{P}_{1}=\frac{-1}{L_{1}^{2}}\left(\alpha \mathbf{I}+\boldsymbol{t}_{12}-2 \alpha \boldsymbol{t}_{11}\right) \\
& \mathbf{P}_{2}=\frac{-1}{L_{2}^{2}}\left(\alpha \mathbf{I}+\boldsymbol{t}_{21}-2 \alpha \boldsymbol{t}_{22}\right) \\
& \mathbf{P}_{3}=\frac{1}{L_{1} L_{2}}\left(\mathbf{I}-\boldsymbol{t}_{11}-\boldsymbol{t}_{22}+\alpha \boldsymbol{t}_{12}\right) \\
& \mathbf{P}_{4}=\frac{1}{L_{1} L_{2}}\left(\mathbf{I}-\boldsymbol{t}_{11}-\boldsymbol{t}_{22}+\alpha \boldsymbol{t}_{21}\right)
\end{aligned}
$$

To calculate $\Delta B$, we can use $B=\sin \theta$, obtaining

$$
\Delta B=\Delta(\sin \theta)=\cos \theta \Delta \theta=\alpha \Delta g_{\mathrm{R}}
$$

Substituting Eqs. (27) and (32) into Eq. (24), we get

$$
\Delta \delta g_{\mathrm{R}}=\frac{-1}{\sqrt{1-\alpha^{2}}} \delta \boldsymbol{u}^{\mathrm{T}} \mathbf{Q}_{1} \Delta \boldsymbol{u}-\frac{\alpha}{\sqrt{1-\alpha^{2}}} \delta g_{\mathrm{R}} \Delta g_{\mathrm{R}}
$$

Using Eqs. (19) and (20), we get $\delta g_{\mathrm{R}} \Delta g_{\mathrm{R}}$ as follows:

$$
\delta g_{\mathrm{R}} \Delta g_{\mathrm{R}}=\delta \boldsymbol{u}^{\mathrm{T}} \mathbf{G}^{\mathrm{T}} \mathbf{G} \Delta \boldsymbol{u}=\frac{1}{1-\alpha^{2}} \delta \boldsymbol{u}^{\mathrm{T}}
$$




$$
\left[\begin{array}{rrrr}
\mathbf{P}_{5} & -\mathbf{P}_{5} & \mathbf{P}_{7} & -\mathbf{P}_{7} \\
-\mathbf{P}_{5} & \mathbf{P}_{5} & -\mathbf{P}_{7} & \mathbf{P}_{7} \\
\mathbf{P}_{8} & -\mathbf{P}_{8} & \mathbf{P}_{6} & -\mathbf{P}_{6} \\
-\mathbf{P}_{8} & \mathbf{P}_{8} & -\mathbf{P}_{6} & \mathbf{P}_{6}
\end{array}\right] \Delta \boldsymbol{u}
$$

where $\mathbf{P}_{5}, \mathbf{P}_{6}, \mathbf{P}_{7}$, and $\mathbf{P}_{8}$ are given by

$\mathbf{P}_{5}=\frac{1}{L_{1}^{2}}\left(\boldsymbol{t}_{22}-\alpha \boldsymbol{t}_{12}-\alpha \boldsymbol{t}_{21}+\alpha^{2} \boldsymbol{t}_{11}\right)$

$\mathbf{P}_{6}=\frac{1}{L_{2}^{2}}\left(\boldsymbol{t}_{11}-\alpha \boldsymbol{t}_{12}-\alpha \boldsymbol{t}_{21}+\alpha^{2} \boldsymbol{t}_{22}\right)$

$\mathbf{P}_{7}=\frac{1}{L_{1} L_{2}}\left(\boldsymbol{t}_{21}-\alpha \boldsymbol{t}_{11}-\alpha \boldsymbol{t}_{22}+\alpha^{2} \boldsymbol{t}_{12}\right)$

$\mathbf{P}_{8}=\frac{1}{L_{1} L_{2}}\left(\boldsymbol{t}_{12}-\alpha \boldsymbol{t}_{11}-\alpha \boldsymbol{t}_{22}+\alpha^{2} \boldsymbol{t}_{21}\right)$

Finally, substituting Eq. (34) into Eq. (33), we get

$$
\begin{aligned}
\Delta \delta g_{\mathrm{R}} & =\delta \boldsymbol{u}^{\mathrm{T}}\left(\frac{-1}{\sqrt{1-\alpha^{2}}} \mathbf{Q}_{1}+\frac{-\alpha}{\sqrt{1-\alpha^{2}}} \mathbf{G}^{\mathrm{T}} \mathbf{G}\right) \Delta \boldsymbol{u} \\
& =\delta \boldsymbol{u}^{\mathrm{T}} \mathbf{Q} \Delta \boldsymbol{u}
\end{aligned}
$$

Using the results obtained in Eqs. (19), (20), and (39), and substituting into Eq. (11), we get the following tangent stiffness matrix for the rotational constraint, using the Lagrange multiplier method:

$\mathbf{K}_{\mathrm{R}}=\left[\begin{array}{cc}\lambda_{\mathrm{R}} \mathbf{Q} & \mathbf{G}^{\mathrm{T}} \\ \mathbf{G} & \mathbf{0}\end{array}\right]$

To derive the tangent stiffness matrix of the rotational constraint using a penalty stiffness method, we substitute Eqs. (34) and (39) into Eq. (7) to get the following equation:

$\mathbf{K}_{\mathrm{R}}=\varepsilon_{\mathrm{R}}\left(\mathbf{G}^{\mathrm{T}} \mathbf{G}+g_{\mathrm{R}} \mathbf{Q}\right)$.

\subsection{Method 2: Non-consistent Approach}

In this approach, in contrast to the consistent formulation presented above, we do not assume a particular form of the shape functions for the beam elements. Instead we assume that the contact normal and contact location are constant (independent of the deformations) in a single iteration. However, the contact normal and location can (and most often do) vary from one iteration to the next. For this reason we consistently update them between iterations. Using the assumption of independence of contact normal and location from the deformations (in a single iteration), the contribution from the change of contact normal to the linearization of the contact gap function vanishes. This simplifies the formulation significantly. Also, we will use a penalty stiffness method to enforce the rotational constraint. Using these assumptions we can write the incremental change of the rotational gap, $\Delta g_{\mathrm{R}}$, as

$\Delta g_{\mathrm{R}}=\Delta \theta_{2 \mathrm{~N}}-\Delta \theta_{1 \mathrm{~N}}$

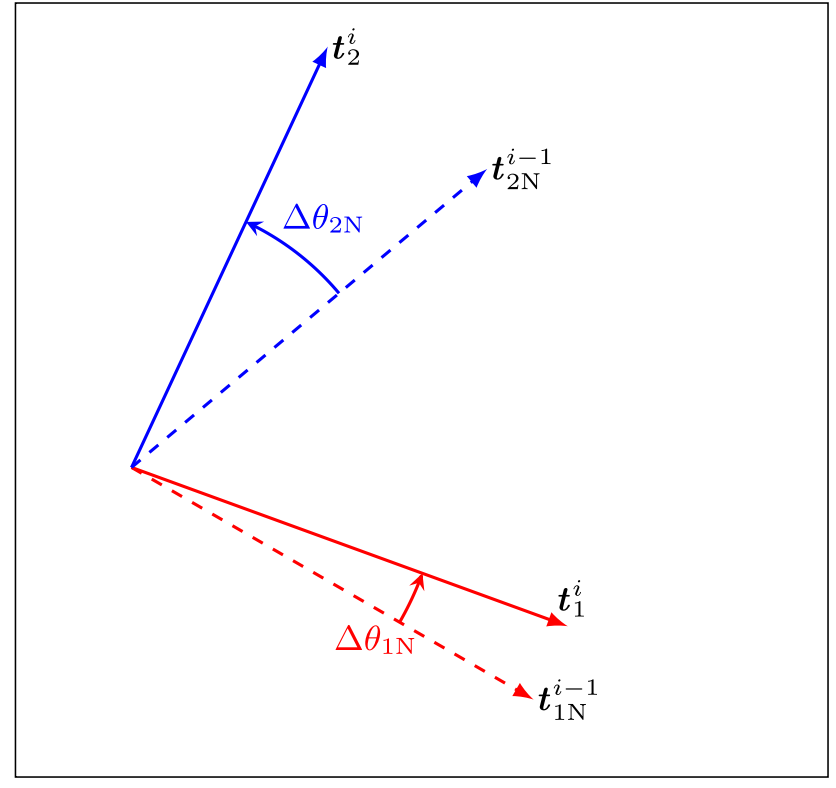

Figure 2. Projection of the change in the rotational degree of freedom of the beams, $\Delta \boldsymbol{\theta}_{1}$ and $\Delta \boldsymbol{\theta}_{2}$, at the contact point, onto the plane of the current contact normal $\boldsymbol{n}$. The superscripts $i$ and $i-1$ refer to iteration numbers. (The view of the figure is along the contact normal.)

where $\Delta \theta_{1 \mathrm{~N}}$ and $\Delta \theta_{2 \mathrm{~N}}$ are shown in Fig. 2. In this figure, the superscripts $i-1$ and $i$ are iteration numbers and the subscript $\mathrm{N}$ refers to the projection onto the plane of the current contact normal. $\boldsymbol{t}_{j}$ is the unit tangent vector to Beam $j$ and $\boldsymbol{\theta}_{j}$ is the rotation vector of Beam $j$ at the contact point $(j=1,2)$. We can observe that $\Delta \theta_{1 \mathrm{~N}}$ and $\Delta \theta_{2 \mathrm{~N}}$ are in fact projections of the changes in the rotation vectors of Beams 1 and 2 at the contact point, onto the plane of current contact normal (the plane spanned by the tangents to the beams at the contact point), respectively:

$\left\{\begin{array}{l}\Delta \theta_{1 \mathrm{~N}}=\left(\theta_{1 \mathrm{~N}}^{i}-\theta_{1 \mathrm{~N}}^{i-1}\right)=\left(\boldsymbol{\theta}_{1}^{i}-\boldsymbol{\theta}_{1}^{i-1}\right) \cdot \boldsymbol{n}=\Delta \boldsymbol{\theta}_{1} \cdot \boldsymbol{n} \\ \Delta \theta_{2 \mathrm{~N}}=\left(\theta_{1 \mathrm{~N}}^{i}-\theta_{2 \mathrm{~N}}^{i-1}\right)=\left(\boldsymbol{\theta}_{2}^{i}-\boldsymbol{\theta}_{2}^{i-1}\right) \cdot \boldsymbol{n}=\Delta \boldsymbol{\theta}_{2} \cdot \boldsymbol{n}\end{array}\right.$

where $\Delta \boldsymbol{\theta}_{1}$ and $\Delta \boldsymbol{\theta}_{2}$ are the incremental changes in the rotation vectors of Beams 1 and 2 evaluated at the contact point and $\boldsymbol{n}$ is the current contact normal which is updated at each iteration. $\Delta \boldsymbol{\theta}_{1}$ and $\Delta \boldsymbol{\theta}_{2}$ can be related to the nodal incremental displacements of Beams 1 and 2, $\Delta \boldsymbol{u}_{1}$ and $\Delta \boldsymbol{u}_{2}$, through the rotational shape functions of the beam elements, $\mathbf{H}_{\mathrm{R}_{1}}$ and $\mathbf{H}_{\mathrm{R}_{2}}$ :

$\left\{\begin{aligned} \Delta \boldsymbol{\theta}_{1} & =\mathbf{H}_{\mathrm{R}_{1}} \Delta \boldsymbol{u}_{1} \\ \Delta \boldsymbol{\theta}_{2} & =\mathbf{H}_{\mathrm{R}_{2}} \Delta \boldsymbol{u}_{2}\end{aligned}\right.$

The present derivation is independent of the beam formulation, however, as examples, the rotational shape functions are derived in Appendices B1 and B2 for a linear and an EulerBernoulli beam element, respectively. Substituting Eqs. (43) 
and (44) into Eq. (42), we obtain

$$
\Delta g_{\mathrm{R}}=\left[\begin{array}{ll}
-\boldsymbol{n}^{\mathrm{T}} & \boldsymbol{n}^{\mathrm{T}}
\end{array}\right] \mathbf{H}_{\mathrm{R}} \Delta \boldsymbol{u}
$$

where $\mathbf{H}_{\mathrm{R}}$ and $\Delta \boldsymbol{u}$ are given by

$$
\begin{aligned}
\mathbf{H}_{\mathrm{R}} & =\left[\begin{array}{cc}
\mathbf{H}_{\mathrm{R}_{1}} & \mathbf{0} \\
\mathbf{0} & \mathbf{H}_{\mathrm{R}_{2}}
\end{array}\right] \\
\Delta \boldsymbol{u} & =\left[\begin{array}{l}
\Delta \boldsymbol{u}_{1} \\
\Delta \boldsymbol{u}_{2}
\end{array}\right]
\end{aligned}
$$

Similarly,

$$
\delta g_{\mathrm{R}}=\delta \boldsymbol{u} \mathbf{H}_{\mathrm{R}}^{\mathrm{T}}\left[\begin{array}{c}
-\boldsymbol{n} \\
\boldsymbol{n}
\end{array}\right]
$$

Based on the assumption made for this approach, that the contact normal and location are independent of the nodal displacements in an iteration, we obtain

$$
\Delta \delta g_{\mathrm{R}}=0
$$

Using Eqs. (7), (45), (48), and (49), we can write the tangent stiffness matrix for the rotational constraint as

$\mathbf{K}_{\mathrm{R}}=\varepsilon_{\mathrm{R}} \mathbf{H}_{\mathrm{R}}^{\mathrm{T}} \mathbf{N} \mathbf{H}_{\mathrm{R}}$

where $\mathbf{N}$ is

$\mathbf{N}=\left[\begin{array}{rr}n \otimes n & -n \otimes n \\ -n \otimes n & n \otimes n\end{array}\right]=\left[\begin{array}{c}-n \\ n\end{array}\right]\left[\begin{array}{ll}-n^{\mathrm{T}} & n^{\mathrm{T}}\end{array}\right]$

Using Eq. (48), the internal force vector of this rotational constraint element can be written as

$\mathbf{F}_{\mathrm{R}_{\mathrm{int}}}=\varepsilon_{\mathrm{R}} g_{\mathrm{R}} \mathbf{H}_{\mathrm{R}}^{\mathrm{T}}\left[\begin{array}{c}-\boldsymbol{n} \\ \boldsymbol{n}\end{array}\right]$

\section{Numerical Examples}

In this section, we verify the correctness and benchmark performance of the suggested methods by solving different examples. In the first example, we consider a case where the points on each beam, corresponding to the contact point, do not tend to move in the normal and tangential directions with respect to each other and only the rotational constraint acts. We will compare the results with an ideal case in which a common node exists instead of the contact point. The goal of this example is to evaluate the correctness and performance of the suggested formulations. In the second example, we consider a case where all the normal, tangential, and rotational constraints are active. Here we can compare the performance of the two suggested formulations, as well as, evaluate the effect of including the rotational constraint. In the third example, a small structure consisting of intertwined beams is considered. The main focus of this example is to study how inclusion of the rotational constraint and addition of extra beams to the structure affect the stiffness of the whole structure. In the last example, we analyze a random network of interconnected fibers with and without rotational constraint. The selection of this fourth example is motivated by the application to the study of the strength and stiffness of 3-D fiber networks, where the fibers may have multiple contact points along their length and the corresponding simulations will benefit from the presented formulation.

In the first, second, and third examples, we consider beams with a circular cross-section with a radius of 0.25 , a length of 10 (and 5 in the third example), and an elasticity modulus of $10^{6}$, all in consistent units. The applied forces and displacements are in the same consistent units as well. In the fourth example, a random network is analyzed where the length and the radii of the cross-sections of the beams are chosen randomly within a prescribed interval.

The proposed formulations are implemented in MATLAB R2015a ${ }^{1}$ and used to analyze Examples 1 and 2. Example 3 and 4 are analyzed using an in-house implementation of the non-consistent formulation (Sect. 3.2) in Fortran.

\subsection{Example 1}

In this example we consider two crossing perpendicular beams. The translational degrees of freedom are constrained at the two ends of one beam, while the other beam is given a prescribed in-plane displacement at the two ends, which would cause a rigid body rotation of $15^{\circ}$ around the midpoint of the beam if the rotational constraint were not present. The initial configuration and boundary conditions are shown in Fig. 3a. We have no out-of-plane deformation and we assume bonded constraint (inseparable contact) between the beams. A sample deformed configuration is shown in Fig. $3 b$. To crosscheck the results, we consider another case, where the beams are located in the same plane and have a common node instead of the contact point. Although these two problems are not exactly the same, as the beams inter-penetrate in the case of a shared node, the in-plane displacements should be similar and we will have a comprehension of the convergence rate, which we can use as a reference. In Fig. 4, the deformed shape for the case with the common node is compared with the deformed shape for the case with the rotational constraint element, when using a rotational penalty stiffness of $10^{5}$. It should be noted that although the deformations of the two cases are similar, the configurations are different: in the case with a contact point, the beams are located at different $z$ levels, whereas in the case with the common node, they are in the same plane. The effect of the size of the rotational penalty stiffness on the rotational gap is shown in Fig. 5. Note that the common node is similar to a contact point with an infinitely large penalty stiffness, which results in a zero

\footnotetext{
${ }^{1}$ The code and examples can be downloaded from: www.mathworks.com/matlabcentral/fileexchange/ 55723-beam2beam
} 


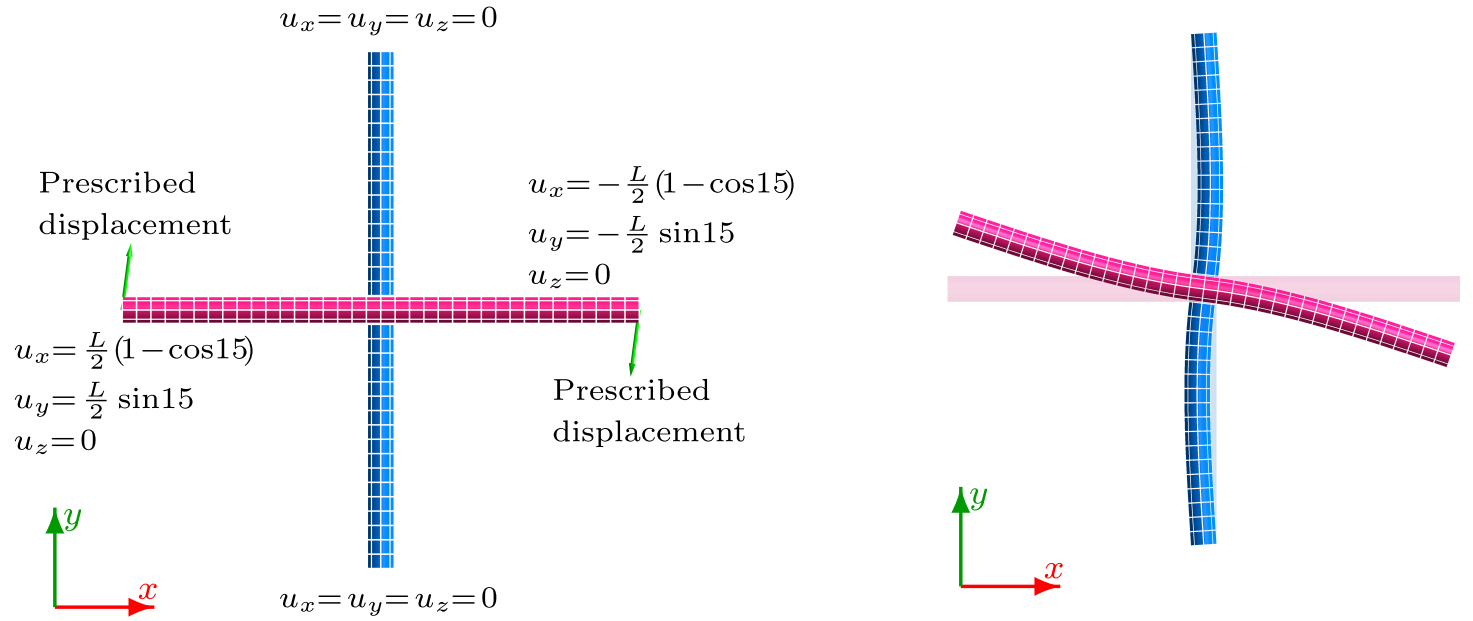

(a)

(b)

Figure 3. (a) Initial geometry and boundary conditions, and (b) a sample deformed shape for Example 1. (Wireframe is for visualization purposes only and does not represent meshing.)

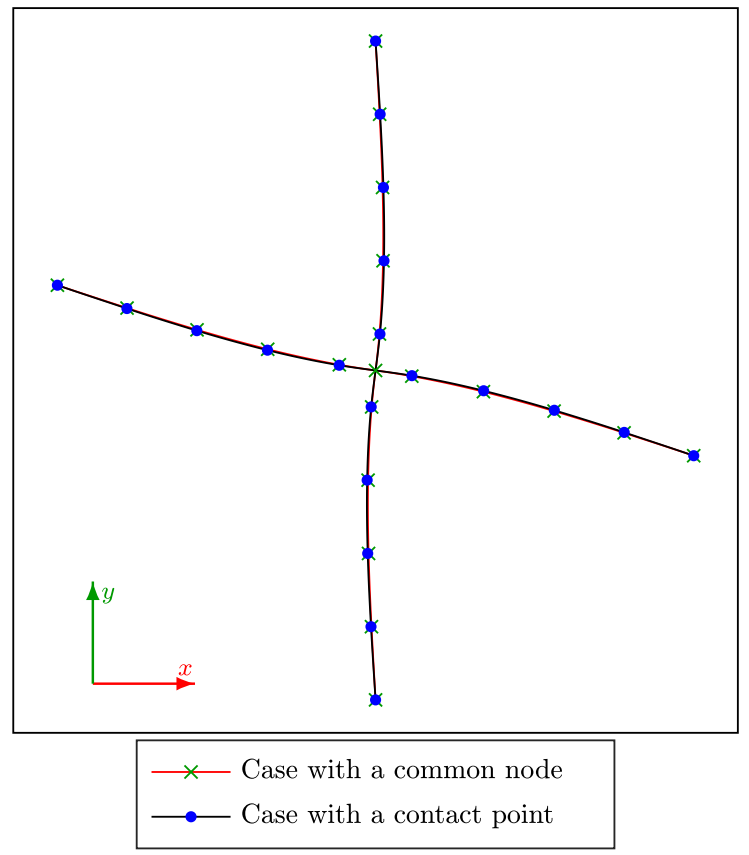

Figure 4. Comparison of the deformed shape of beams with a contact point versus beams connected over a common node.

gap. The change in the imbalance of energy during the analysis is shown in Fig. 6 for the case with the common node and the case with the contact point using both consistent and non-consistent formulations. As can be seen, including the rotational constraint has a small effect on the convergence of the solution. Due to the fact that the contact point and normal remain unchanged during the iterations of this analysis,

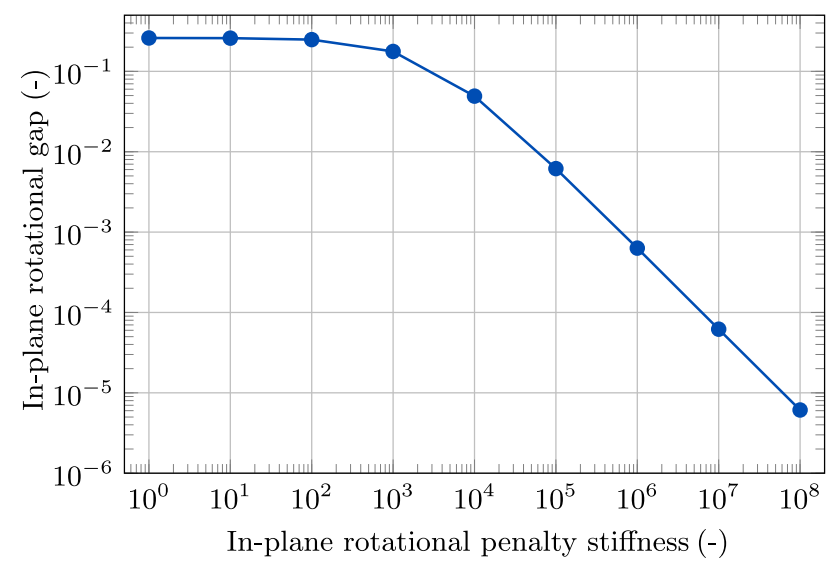

Figure 5. Effect of rotational penalty stiffness on the rotational gap.

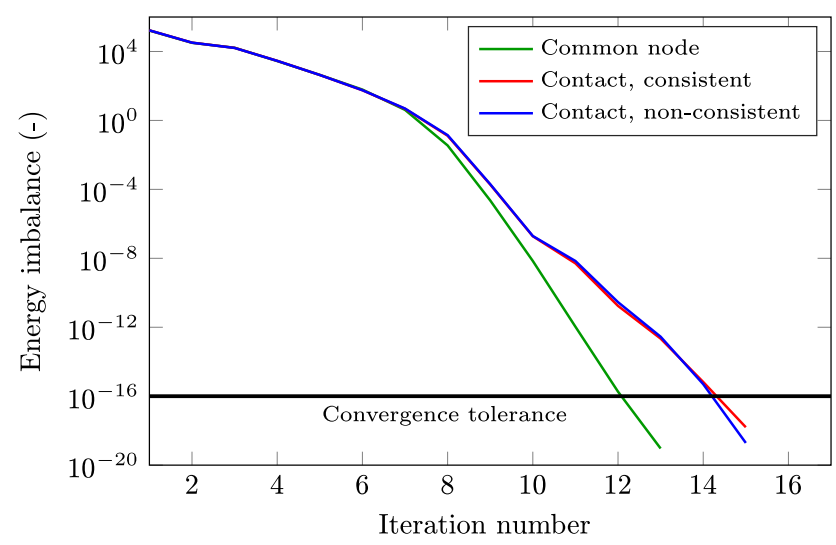

Figure 6. Energy imbalance reduction during the analysis of Example 1 . 

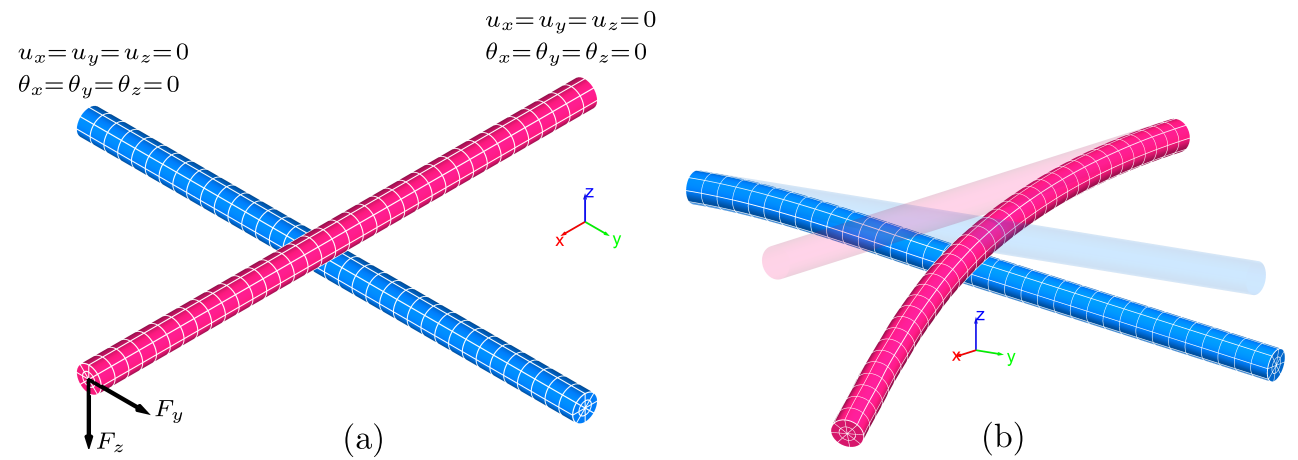

Figure 7. (a) Initial geometry and boundary conditions, and (b) a sample deformed shape for Example 2. (Wireframe is for visualization purposes only and does not represent meshing.)

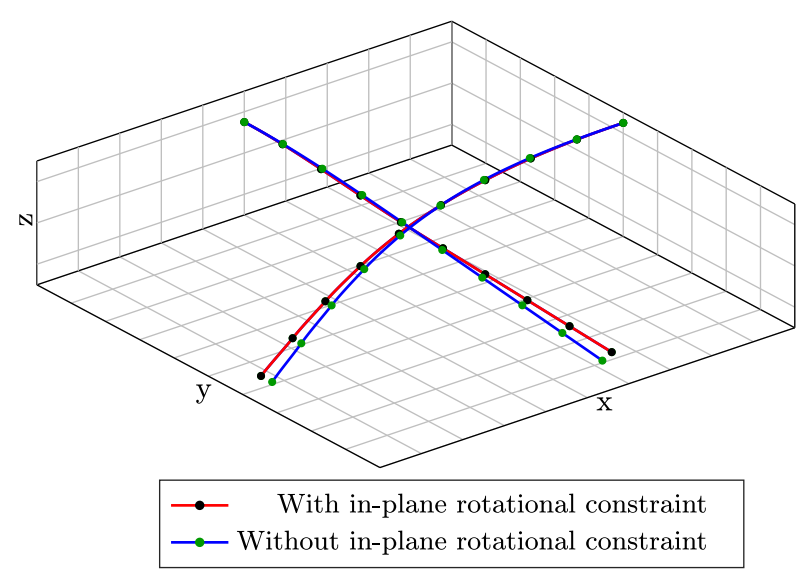

Figure 8. Comparison of the deformed shape of beams with and without the rotational constraint in Example 2.

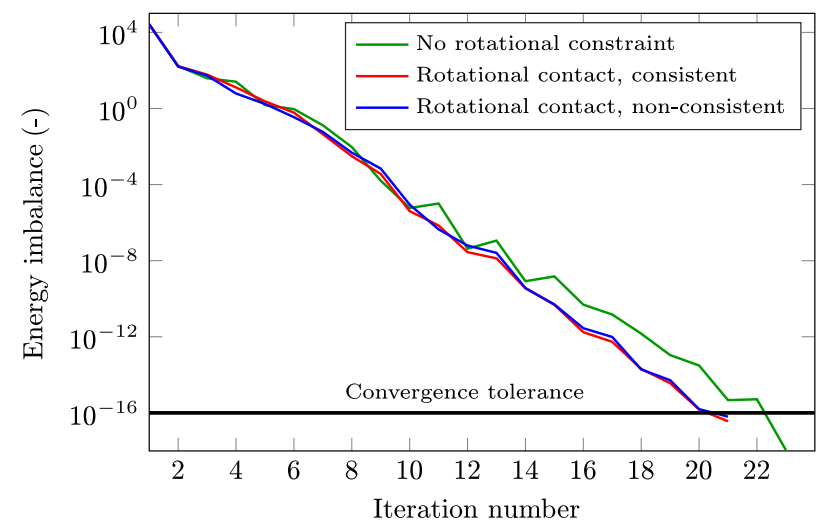

Figure 9. Energy imbalance reduction during the analysis of Example 2 .

the proposed consistent and non-consistent methods behave identically in solving this example.

\subsection{Example 2}

In this example, we consider two crossing perpendicular beams which are fully constrained at one end. We apply an in-plane force of $F_{y}=50$ units and an out-of-plane force of $F_{z}=-50$ units to the free end of the upper beam, as shown in Fig. 7a. We assume a bonded constraint (inseparable contact) between the beams and analyze the problem with and without the rotational constraint. The deformed configuration of the beams is shown in Fig. 7b and in Fig. 8 the deformed shapes of the beams, including and ignoring the rotational constraint, are compared.

The change in the energy imbalance during the solution process is shown in Fig. 9 when using consistent and nonconsistent formulations, and when ignoring the rotational constraint. We can see that including or ignoring the rotational constraint has a small effect on the convergence. It should also be noted that the consistent and non-consistent formulations both lead to the same results for nodal deformations and forces. The consistent method has a better convergence if very narrow tolerances are considered and the non-consistent method has better stability with larger load steps.

\subsection{Example 3}

In this example, we start with a structure consisting of 4 intertwined beams of length 10, as shown in Fig. 10a. The endpoints of the beams at the left side of the structure are fixed to avoid translation and a unit displacement $\Delta=1$ is applied to the endpoints located at the right side of the structure, as shown in Fig. 10b. The stiffness of the structure is calculated as follows:

$k=-\frac{R}{\Delta}$

where $R$ is the sum of the reaction forces in the $x$ direction (direction of the prescribed displacement) collected from the constrained nodes on the left side, and $\Delta$ is the unit displace- 


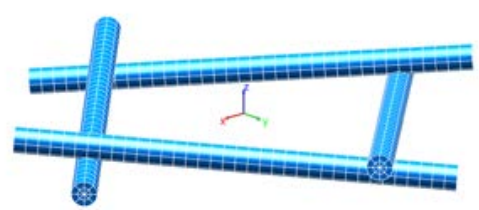

(a)

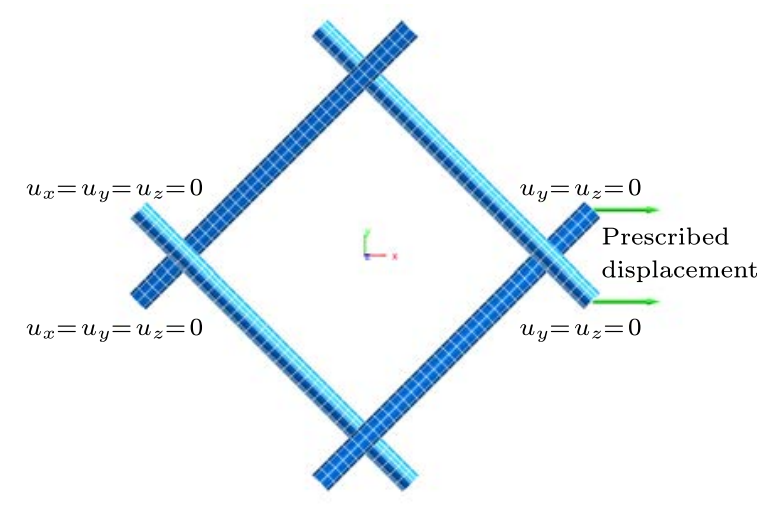

(b)

Figure 10. (a) The intertwined geometry (b) the applied boundary conditions for Example 3. (Wireframe is for visualization purposes only and does not represent meshing.)

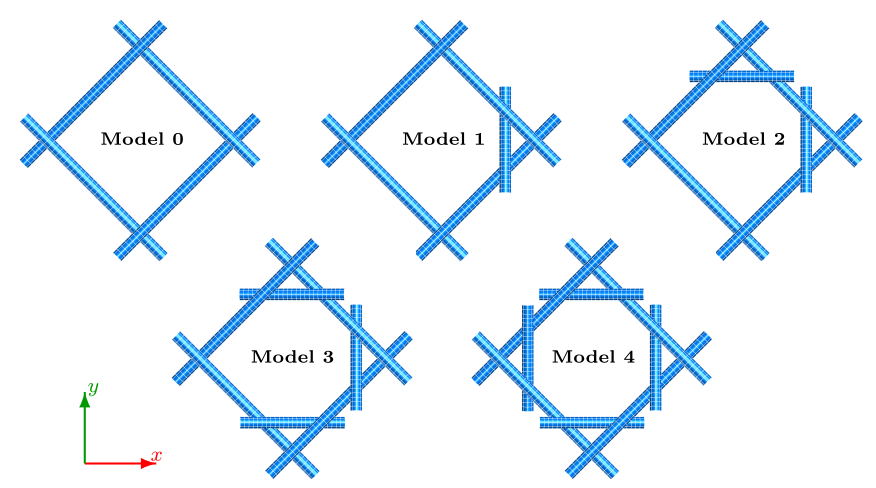

Figure 11. Different models used in Example 3.

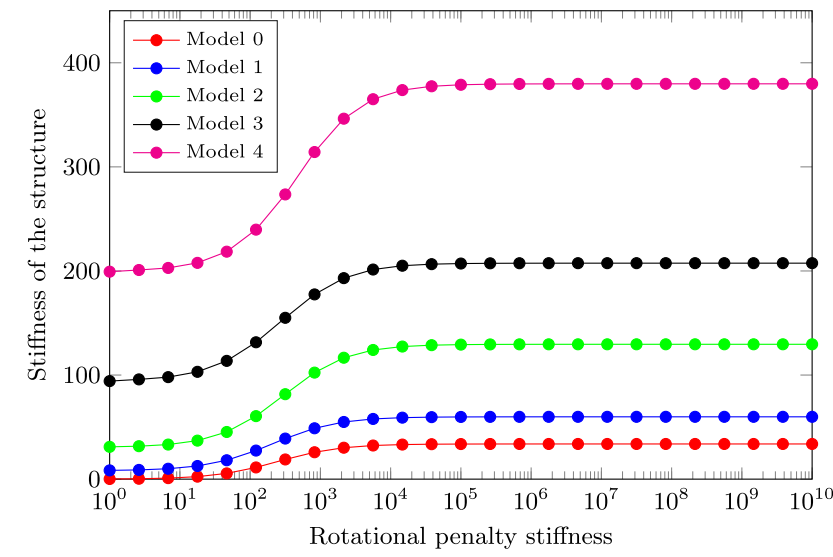

Figure 12. Effect of rotational penalty stiffness on the stiffness of different models of Example 3. ment applied on the right side. Without the rotational constraints the structure has zero stiffness and undergoes rigid body rotations.

We then modified our model, by adding beams of length 5 that passed through the intertwined beams in order to get the models shown in Fig. 11. To study the effect of the rotational penalty stiffness on the stiffness of the structure, we included the rotational constraint and changed the rotational penalty stiffness. The results are shown in Fig. 12 for the different models of Fig. 11). As can be seen in this figure, if the rotational penalty stiffness is above a certain value $\left(10^{4}\right.$ in this example) the results are not affected. Figure 13a shows the structure stiffness of the different models, with and without the rotational constraint. In Fig. 13b the ratio between the stiffness of the models when including and excluding the rotational constraint is plotted. As can be seen, with increasing the number of beams and bonds between them, this ratio decreases, however, its effect is considerable even in the last case (Model 4). In the next example, we will demonstrate that even with a random network consisting of hundreds of fibers, the rotational constraint has a big effect on the stiffness. 


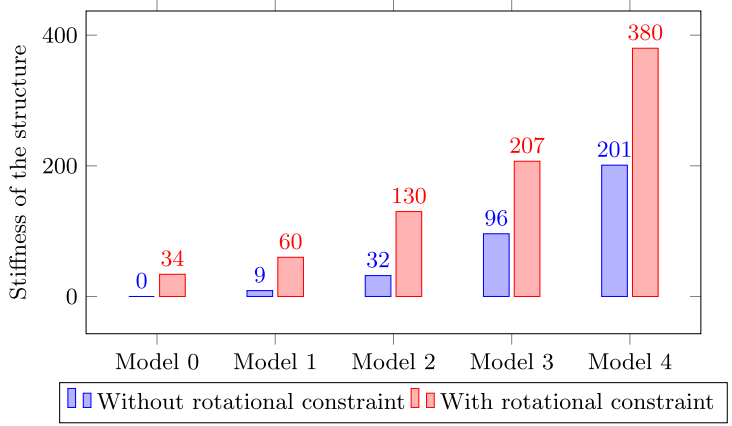

(a)

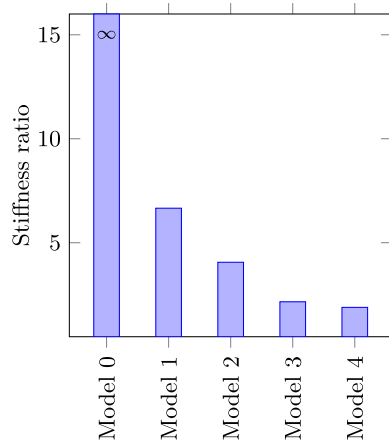

(b)

Figure 13. Effect of rotational penalty stiffness on the stiffness of different models of Example 3. (a) Structural stiffness values, (b) ratio between structural stiffness with and without rotational constraint.

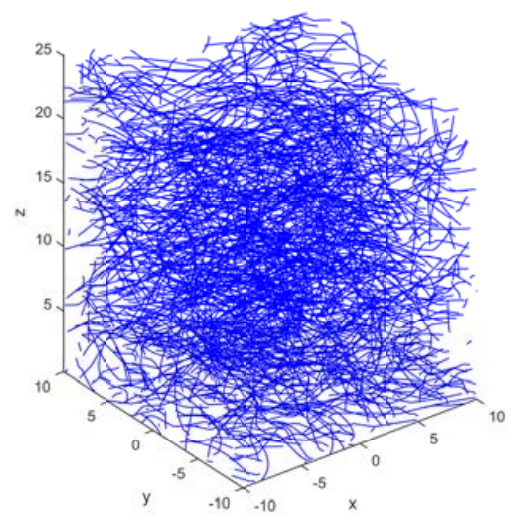

(a)

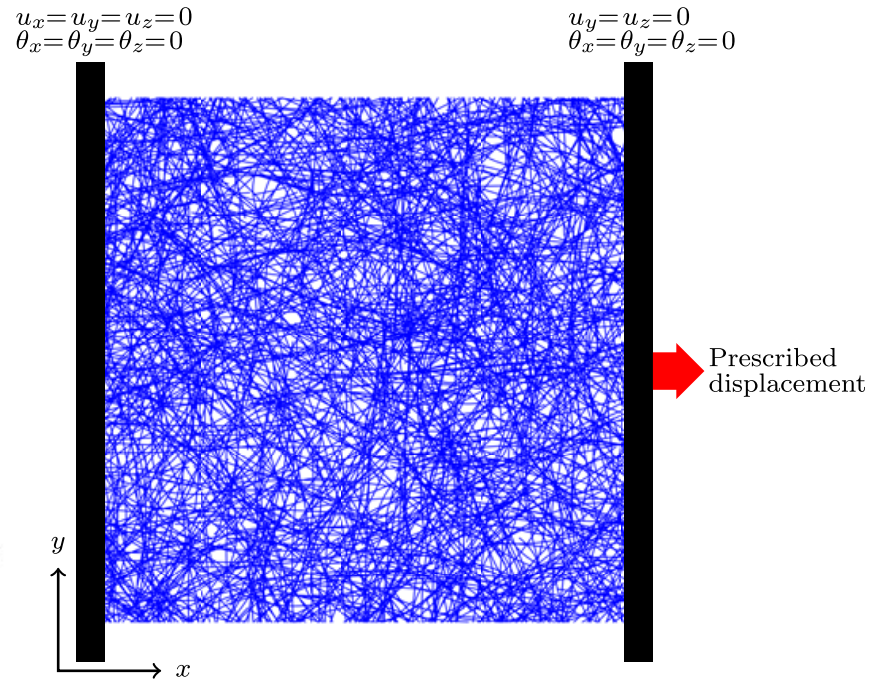

(b)

Figure 14. Initial configuration of the random network of Example 3. (a) 3-D configuration, and (b) top view boundary conditions.

\subsection{Example 4}

In this example, we generated a random fiber network of size $20 \times 20$ units, using a deposition technique (Kulachenko and Uesaka, 2012). The network consists of 776 fibers meshed with 11789 beam elements of equal length coming into contact at 1322 points. An initial inter-penetration which happened between certain beams was resolved by offsetting the contact distance by the initial value of penetration. The mean, min, max and standard deviations for the fiber lengths and cross-sectional radii are given in Table (1). It should be noted that the width of the network is larger than the length of the longest fiber and as a result, no fibers are extending from one side of the network to the other. We have defined 20 different materials with equally spaced values of the elasticity
Table 1. Geometric data of the random networks.

\begin{tabular}{lrrrr}
\hline & Mean & SD $^{*}$ & Min & Max \\
\hline Length (-) & 10 & 3 & 5 & 15 \\
Radius of cross-section (-) & 0.25 & 0.03 & 0.2 & 0.3 \\
\hline
\end{tabular}

* Standard deviation

modulus, in the range of $5 \times 10^{5}$ to $1.5 \times 10^{6}$, and randomly assigned one of these materials to each fiber.

All the contact points are assumed to be inseparable (bonded constraints) throughout the analysis. We assumed that no new contacts are established during the deformation. A total prescribed displacement of 3 units corresponding to 


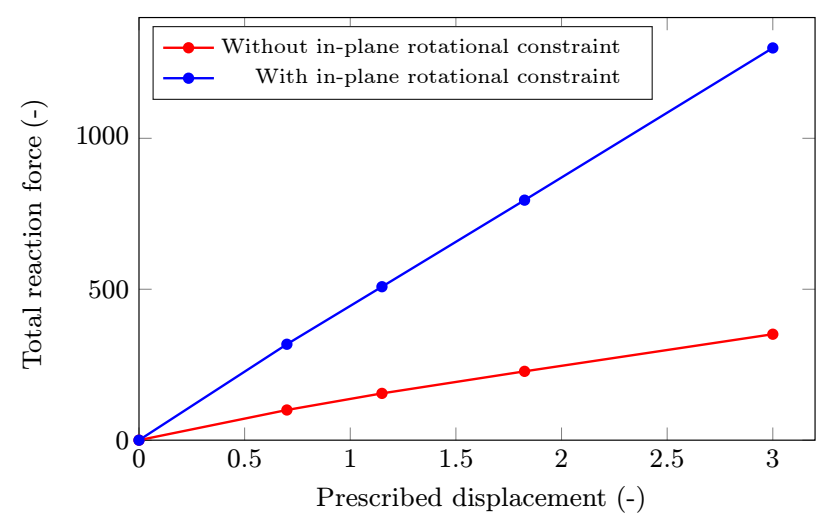

Figure 15. Total reaction force versus prescribed displacement in Example 3, with and without rotational constraint.

$15 \%$ of the network length is applied to the model, as shown in Fig. 14b. The 3-D configuration of the network is shown in Fig. 14a. To demonstrate how the rotational constraint affects the stiffness of the network, we performed a geometrically non-linear analysis with and without the rotational constraint. The sum of the reaction forces in the direction of the applied displacement is plotted versus the applied displacement for both cases in Fig. 15. In both case, the initial response was linear. However, the inclusion of the rotational constraint increases the stiffness by a factor of 3.5 approximately. This contribution from the rotational constraint is dependent on the geometry of network, for example with a more compact network (with more bonds between fibers and shorter distance between contact points along a fiber), the effect of the rotational constraint decreases. It is worth mentioning that the problem computed with rotational constraints required significantly fewer iterations, as the added constraints effectively prevented any rigid body rotations of the loosely constrained fibers.

\section{Summary and Conclusions}

Two alternative methods were suggested in order to handle the rotational constraint between beam elements. In the consistent method, we followed the derivation methodology encountered in earlier works dealing with normal and frictional contact between beams. This formulation generally requires an assumption for the shape functions. For the sake of simplicity, we assumed linear shape functions for the underlying beam elements. The non-consistent method does not require predefined shape functions for the beam elements, as it assumes that the contact normal is independent of the nodal deformations in each iteration (the change of contact normal at each iteration does not contribute to the linearization of the gap function and as a result to the tangent stiffness matrix), and it is updated between iterations. Despite large differences in their mathematical formulations, the two methods generally show similar convergence rates and stability for the chosen examples. However, the non-consistent method offers the advantages of simpler derivations and an easier implementation. In the considered examples where the beams are well constrained, the inclusion of the rotational constraint does not generally affect the convergence rate of the problems.

Including the rotational constraints in the problem of a random fiber network significantly affected the stiffness of the network, due to the addition of constraints in the system. It clearly shows that ignoring rotational constrains can yield inappropriate results in such cases. In addition, the presence of rotational constraints improves the stability of the non-linear solution procedure by effectively preventing rigid body rotation, in particular, in the fibers which have few contacts along their length. 


\section{Appendix A: Mathematical Derivations}

\section{A1 Variation of a unit vector}

If $\boldsymbol{x}_{i}$ and $\boldsymbol{x}_{j}$ are the positions of two points in 3-D space, the unit vector $v$ along the line connecting a point at $x_{1}$ to a point at $\boldsymbol{x}_{j}$ is

$v=\frac{x_{j}-x_{i}}{L}$

where $L$ is the distance between the two points,

$$
L=\left\|x_{j}-x_{i}\right\|=\sqrt{\left(x_{j}-x_{i}\right)^{2}}
$$

The variation of $\boldsymbol{v}$ can be written in terms of the variations of the locations of the endpoints, $\delta \boldsymbol{x}_{i}$ and $\delta \boldsymbol{x}_{j}$, and the variation of the distance between the points, $\delta L$, as follows:

$\delta \boldsymbol{v}=\frac{L \delta\left(\boldsymbol{x}_{j}-\boldsymbol{x}_{i}\right)-\left(\boldsymbol{x}_{j}-\boldsymbol{x}_{i}\right) \delta L}{L^{2}}$

The variation of the distance between the endpoints, $\delta L$, can also be written in terms of the variation of the locations of the endpoints:

$\delta L=\frac{1}{L}\left(\boldsymbol{x}_{j}-\boldsymbol{x}_{i}\right) \cdot \delta\left(\boldsymbol{x}_{j}-\boldsymbol{x}_{i}\right)$.

The variation of the locations of the endpoints can be calculated as follows:

$\delta \boldsymbol{x}_{a}=\delta\left(\boldsymbol{X}_{a}+\boldsymbol{u}_{a}\right)=\delta \boldsymbol{u}_{a} \quad(a=i, j)$

where $\boldsymbol{X}_{a}$ is the initial position and $\boldsymbol{u}_{a}$ is the displacement of point $a(a=i, j)$. Substituting Eqs. (A4), (A5) into Eq. (A3) we get the variation of the unit vector $v$ in terms of the variations of the displacements of the endpoints, $\delta \boldsymbol{u}_{i}$ and $\delta \boldsymbol{u}_{j}$

$\delta \boldsymbol{v}=\frac{1}{L}(\mathbf{I}-\boldsymbol{v} \otimes \boldsymbol{v}) \cdot\left(\delta \boldsymbol{u}_{j}-\delta \boldsymbol{u}_{i}\right)$

\section{A2 Linearization of the variation of a unit vector}

We consider the unit vector $v$ introduced in Appendix (A1) and linearize the variation of this vector, $\delta \boldsymbol{v}$, given in Eq. (A6):

$$
\begin{aligned}
\Delta \delta \boldsymbol{v}= & \frac{1}{L} \Delta(\mathbf{I}-\boldsymbol{v} \otimes \boldsymbol{v}) \cdot\left(\delta \boldsymbol{u}_{j}-\delta \boldsymbol{u}_{i}\right) \\
& +\frac{1}{L}(\mathbf{I}-\boldsymbol{v} \otimes \boldsymbol{v}) \cdot \Delta\left(\delta \boldsymbol{u}_{j}-\delta \boldsymbol{u}_{i}\right) \\
& -\frac{1}{L^{2}}(\mathbf{I}-\boldsymbol{v} \otimes \boldsymbol{v}) \cdot\left(\delta \boldsymbol{u}_{j}-\delta \boldsymbol{u}_{i}\right) \Delta L
\end{aligned}
$$

We know that $\delta \boldsymbol{u}_{a}(a=i, j)$ is independent of $\boldsymbol{u}_{a}(a=i, j)$. This means that $\Delta \delta \boldsymbol{u}_{a}=0(a=i, j)$, and as a result, the second term in Eq. (A7) will be zero:

$$
\frac{1}{L}(\mathbf{I}-\boldsymbol{v} \otimes \boldsymbol{v}) \cdot \Delta\left(\delta \boldsymbol{u}_{j}-\delta \boldsymbol{u}_{i}\right)=0 .
$$

The linearization of the distance between the end-points is calculated in the same way as its variation, which is given in Eq. (A4):

$$
\Delta L=\frac{1}{L}\left(x_{j}-x_{i}\right) \cdot \Delta\left(x_{j}-x_{i}\right)=v \cdot \Delta\left(x_{j}-x_{i}\right)
$$

Substituting this equation into the last term of Eq. (A7), we get

$$
\begin{aligned}
\frac{1}{L^{2}} & (\mathbf{I}-\boldsymbol{v} \otimes \boldsymbol{v}) \cdot\left(\delta \boldsymbol{u}_{j}-\delta \boldsymbol{u}_{i}\right) \Delta L \\
& =-\frac{1}{L^{2}}\left((\mathbf{I}-\boldsymbol{v} \otimes \boldsymbol{v}) \cdot\left(\delta \boldsymbol{u}_{j}-\delta \boldsymbol{u}_{i}\right)\right)\left(\boldsymbol{v} \cdot \Delta\left(\boldsymbol{x}_{j}-\boldsymbol{x}_{i}\right)\right) \\
& =-\frac{1}{L^{2}}\left(\left(\delta \boldsymbol{u}_{j}-\delta \boldsymbol{u}_{i}\right) \cdot(\mathbf{I}-\boldsymbol{v} \otimes \boldsymbol{v})^{\mathrm{T}}\right)\left(\boldsymbol{v} \cdot \Delta\left(\boldsymbol{x}_{j}-\boldsymbol{x}_{i}\right)\right) \\
& =-\frac{1}{L^{2}}\left(\left(\delta \boldsymbol{u}_{j}-\delta \boldsymbol{u}_{i}\right) \cdot(\mathbf{I}-\boldsymbol{v} \otimes \boldsymbol{v})\right)\left(\boldsymbol{v} \cdot \Delta\left(\boldsymbol{x}_{j}-\boldsymbol{x}_{i}\right)\right) \\
& =-\frac{1}{L^{2}}\left(\delta \boldsymbol{u}_{j}-\delta \boldsymbol{u}_{i}\right) \cdot((\mathbf{I}-\boldsymbol{v} \otimes \boldsymbol{v}) \cdot \boldsymbol{v}) \cdot \Delta\left(\boldsymbol{x}_{j}-\boldsymbol{x}_{i}\right) \\
& =-\frac{1}{L^{2}}\left(\delta \boldsymbol{u}_{j}-\delta \boldsymbol{u}_{i}\right) \cdot(\boldsymbol{v}-\boldsymbol{v}) \cdot \Delta\left(\boldsymbol{x}_{j}-\boldsymbol{x}_{i}\right) \\
& =0
\end{aligned}
$$

As a result, the linearization of the variation of the unit vector $v$ can be calculated as follows:

$\Delta \delta \boldsymbol{v}=\frac{-1}{L}\left(\delta \boldsymbol{u}_{j}-\delta \boldsymbol{u}_{i}\right) \cdot(\Delta \boldsymbol{v} \otimes \boldsymbol{v}+\boldsymbol{v} \otimes \Delta \boldsymbol{v})$.

\section{A3 Dot product of the linearization of the variation of a unit vector with another unit vector}

We consider the unit vector $v$ introduced in Appendix (A1), and calculate the dot product of $\Delta \delta \boldsymbol{v}$ given in Eq. (A11) with a unit vector $\boldsymbol{w}$ :

$$
\begin{aligned}
\Delta \delta \boldsymbol{v} \cdot \boldsymbol{w} & =\frac{-1}{L}\left(\delta \boldsymbol{u}_{j}-\delta \boldsymbol{u}_{i}\right) \cdot(\Delta \boldsymbol{v} \otimes \boldsymbol{v}+\boldsymbol{v} \otimes \Delta \boldsymbol{v}) \cdot \boldsymbol{w} \\
& =\frac{-1}{L}\left(\delta \boldsymbol{u}_{j}-\delta \boldsymbol{u}_{i}\right) \cdot((\boldsymbol{v} \cdot \boldsymbol{w}) \Delta \boldsymbol{v}+(\boldsymbol{w} \cdot \Delta \boldsymbol{v}) \boldsymbol{v})
\end{aligned}
$$

The linearization of the unit vector $\boldsymbol{v}$ is calculated similarly to its variation given in Eq. (A6):

$\Delta \boldsymbol{v}=\frac{1}{L}(\mathbf{I}-\boldsymbol{v} \otimes \boldsymbol{v}) \cdot\left(\Delta \boldsymbol{u}_{j}-\Delta \boldsymbol{u}_{i}\right)$

Substituting Eq. (A13) into $(\boldsymbol{w} \cdot \Delta \boldsymbol{v}) \boldsymbol{v}$, which is the last term in parentheses in Eq. (A12), we have

$$
\begin{aligned}
(\boldsymbol{w} \cdot \Delta \boldsymbol{v}) \boldsymbol{v} & =\frac{1}{L}\left(\boldsymbol{w} \cdot\left((\mathbf{I}-\boldsymbol{v} \otimes \boldsymbol{v}) \cdot\left(\Delta \boldsymbol{u}_{j}-\Delta \boldsymbol{u}_{i}\right)\right)\right) \cdot \boldsymbol{v} \\
& =\frac{1}{L}((\boldsymbol{v} \otimes \boldsymbol{w}) \cdot(\mathbf{I}-\boldsymbol{v} \otimes \boldsymbol{v})) \cdot\left(\Delta \boldsymbol{u}_{j}-\Delta \boldsymbol{u}_{i}\right)
\end{aligned}
$$

Putting this equation back into Eq. (A12), we have the dot product of $\Delta \delta \boldsymbol{v}$ with the unit vector $\boldsymbol{w}$ :

$\Delta \delta \boldsymbol{v} \cdot \boldsymbol{w}=\frac{-1}{L^{2}}\left(\delta \boldsymbol{u}_{j}-\delta \boldsymbol{u}_{i}\right)$ 


$$
\cdot((\boldsymbol{v} \cdot \boldsymbol{w}) \mathbf{I}+(\boldsymbol{v} \otimes \boldsymbol{w})) \cdot(\mathbf{I}-\boldsymbol{v} \otimes \boldsymbol{v})) \cdot\left(\Delta \boldsymbol{u}_{j}-\Delta \boldsymbol{u}_{i}\right)
$$

which can alternatively be written as follows:

$$
\begin{aligned}
& \Delta \delta \boldsymbol{v} \cdot \boldsymbol{w}=\frac{-1}{L^{2}}\left(\delta \boldsymbol{u}_{j}-\delta \boldsymbol{u}_{i}\right) \\
& \quad \cdot((\boldsymbol{v} \cdot \boldsymbol{w}) \mathbf{I}+(\boldsymbol{v} \otimes \boldsymbol{w})-2(\boldsymbol{v} \cdot \boldsymbol{w}) \boldsymbol{v} \otimes \boldsymbol{v}) \cdot\left(\Delta \boldsymbol{u}_{j}-\Delta \boldsymbol{u}_{i}\right)
\end{aligned}
$$

\section{A4 Dot product of the variation of a unit vector with the linearization of another unit vector}

Consider the unit vector $v$ introduced in Appendix (A1) and the unit vector $\boldsymbol{w}$ along a line connecting the points located at $\boldsymbol{x}_{m}$ and $\boldsymbol{x}_{n}$. We want to calculate $\delta \boldsymbol{v} \cdot \Delta \boldsymbol{w}$, where $\delta \boldsymbol{v}$ is given in Eq. (A6) and $\Delta \boldsymbol{w}$ is given according to Eq. (A13), as follows:

$\frac{1}{l}(\mathbf{I}-\boldsymbol{w} \otimes \boldsymbol{w}) \cdot\left(\Delta \boldsymbol{u}_{n}-\Delta \boldsymbol{u}_{m}\right)$

Here, $l$ is the distance between the endpoints located at $\boldsymbol{x}_{m}$ and $\boldsymbol{x}_{n}$. As a result, $\delta \boldsymbol{v} \cdot \Delta \boldsymbol{w}$ will be

$$
\begin{aligned}
& \delta \boldsymbol{v} \cdot \Delta \boldsymbol{w}=\frac{1}{L l}\left(\delta \boldsymbol{u}_{j}-\delta \boldsymbol{u}_{i}\right) \\
& \quad \cdot(\mathbf{I}-\boldsymbol{v} \otimes \boldsymbol{v}-\boldsymbol{w} \otimes \boldsymbol{w}+(\boldsymbol{v} \cdot \boldsymbol{w}) \boldsymbol{v} \otimes \boldsymbol{w}) \\
& \quad \cdot\left(\Delta \boldsymbol{u}_{n}-\Delta \boldsymbol{u}_{m}\right)
\end{aligned}
$$

\section{Appendix B: Rotational Shape Functions}

In this section, we will derive the rotational shape functions for a linear and an Euler-Bernoulli beam element in their local coordinate system. Rotation matrices are then used to transfer these shape functions to the global coordinate system. Shape functions interpolate the solution between the discrete values at the nodes, as follows:

$$
\left[\begin{array}{c}
u \\
v \\
w
\end{array}\right]=\mathbf{H} \boldsymbol{u}
$$

where $\boldsymbol{u}$ is the nodal displacement vector given below for an element connecting nodes $i$ and $j$ :

$\boldsymbol{u}=\left[\begin{array}{llllllllllll}u_{i} & v_{i} & w_{i} & \theta_{x_{i}} & \theta_{y_{i}} & \theta_{z_{i}} & u_{j} & v_{j} & w_{j} & \theta_{x_{j}} & \theta_{y_{j}} & \theta_{z_{j}}\end{array}\right]^{\mathrm{T}}$

and $\mathbf{H}$ is the matrix of shape functions. Assuming small deformations in the local coordinate system of the element, the bending angles can be calculated as follows:

$\theta_{y}=\frac{\mathrm{d} w}{\mathrm{~d} x}, \quad \theta_{z}=\frac{\mathrm{d} v}{\mathrm{~d} x}$.

The torsional angle can be found using a linear interpolation between the torsional angle of the nodes of the element. However, as it does not contribute to the in-plane rotational contact formulation, we will exclude that from the rotation vector. Using Eqs. (B1) and (B3), we can write the rotation vector of a point on the element as

$[\boldsymbol{\theta}]=\left[\begin{array}{c}0 \\ \theta_{y} \\ \theta_{z}\end{array}\right]=\left(\frac{\mathrm{d}}{\mathrm{d} x}\left[\begin{array}{c}\mathbf{0} \\ \mathbf{H}(:, 3) \\ \mathbf{H}(:, 2)\end{array}\right]\right) \boldsymbol{u}$

where $\mathbf{H}(:, 2)$ and $\mathbf{H}(:, 3)$ refer to the second and third rows of $\mathbf{H}$ respectively, and $\mathbf{0}$ is a row vector of zeros. As a result, the rotational shape functions of the 2-node element in its local coordinate system can be written as

$\mathbf{H}_{\mathrm{R}_{i}}^{\text {local }}=\frac{\mathrm{d}}{\mathrm{d} x}\left[\begin{array}{c}\mathbf{0} \\ \mathbf{H}(:, 3) \\ \mathbf{H}(:, 2)\end{array}\right]$

where $i$ refers to beam element number.

\section{B1 Linear beam elements}

The linear shape functions for a beam element are as follows:

H

$=\left[\begin{array}{llllllllllll}N_{1} & 0 & 0 & 0 & 0 & 0 & N_{2} & 0 & 0 & 0 & 0 & 0 \\ 0 & N_{1} & 0 & 0 & 0 & 0 & 0 & N_{2} & 0 & 0 & 0 & 0 \\ 0 & 0 & N_{1} & 0 & 0 & 0 & 0 & 0 & N_{2} & 0 & 0 & 0\end{array}\right]$

$N_{1}$ and $N_{2}$ can be written in terms of the nodal coordinates of the beam element, $x_{i}$ and $x_{j}$ :

$N_{1}=\frac{x-x_{i}}{x_{j}-x_{i}}=\frac{x-x_{i}}{l^{e}}$,
$N_{2}=\frac{x_{j}-x}{x_{j}-x_{i}}=\frac{x_{j}-x}{l^{e}}$

where $l^{e}$ is the length of the element. Substituting Eqs. (B6) and (B7) into Eq. (B5) results in the following equation for the rotational shape functions of a linear beam element:

$\mathbf{H}_{\mathrm{R}_{i}}^{\text {local }}=\frac{1}{l^{e}}$

$\left[\begin{array}{cccccccccccc}0 & 0 & 0 & 0 & 0 & 0 & 0 & 0 & 0 & 0 & 0 & 0 \\ 0 & 1 & 0 & 0 & 0 & 0 & 0 & -1 & 0 & 0 & 0 & 0 \\ 0 & 0 & 1 & 0 & 0 & 0 & 0 & 0 & -1 & 0 & 0 & 0\end{array}\right]$

where $i$ refers to beam element number.

\section{B2 Euler-Bernoulli beam elements}

The shape functions for an Euler-Bernoulli beam element are given below.

H

(B9)

$=\left[\begin{array}{cccccccccccc}N_{1} & 0 & 0 & 0 & 0 & 0 & N_{2} & 0 & 0 & 0 & 0 & 0 \\ 0 & H_{1} & 0 & 0 & 0 & H_{2} & 0 & H_{3} & 0 & 0 & 0 & H_{4} \\ 0 & 0 & H_{1} & 0 & -H_{2} & 0 & 0 & 0 & H_{3} & 0 & -H_{4} & 0\end{array}\right]$ 
$N_{1}$ and $N_{2}$ are the linear shape functions from Eq. (B7), and $H_{i}(i=1, \ldots, 4)$ are Hermite shape functions:

$$
\left\{\begin{array}{l}
H_{1}=\frac{1}{4}\left(2-3 \xi+\xi^{3}\right) \\
H_{2}=\frac{1}{8} l^{e}\left(1-\xi-\xi^{2}+\xi^{3}\right) \\
H_{3}=\frac{1}{4}\left(2+3 \xi-\xi^{3}\right) \\
H_{4}=\frac{1}{8} l^{e}\left(-1-\xi+\xi^{2}+\xi^{3}\right)
\end{array}\right.
$$

where $l^{e}$ is the length of the element, and $\xi$ is a parameter that changes from -1 to 1 along the element. $\xi$ is related to the nodal coordinates of the beam element, $x_{i}$ and $x_{j}$, as follows:

$\xi=\frac{2}{l^{e}}\left(x-x_{i}\right)-1 \quad x_{i} \leq x \leq x_{j}$

Substituting Eqs. (B9), (B7) and (B10) into Eq. (B5), we have the following equation for the rotational shape functions of an Euler-Bernoulli beam element:

$\mathbf{H}_{\mathrm{R}_{i}}^{\text {local }}=\frac{1}{l^{e}}$

$$
\left[\begin{array}{cccccccccccc}
0 & 0 & 0 & 0 & 0 & 0 & 0 & 0 & 0 & 0 & 0 & 0 \\
0 & 0 & H_{R_{1}} & 0 & -H_{R_{2}} & 0 & 0 & 0 & H_{R_{3}} & 0 & -H_{R_{4}} & 0 \\
0 & H_{R_{1}} & 0 & 0 & 0 & H_{R_{2}} & 0 & H_{R_{3}} & 0 & 0 & 0 & H_{R_{4}}
\end{array}\right]
$$

where $i$ refers to beam element number and

$$
\left\{\begin{aligned}
H_{\mathrm{R}_{1}} & =\frac{3}{2}\left(-1+\xi^{2}\right) \\
H_{\mathrm{R}_{2}} & =\frac{1}{4} l^{e}\left(-1-2 \xi+3 \xi^{2}\right) \\
H_{\mathrm{R}_{3}} & =\frac{3}{2}\left(1-\xi^{2}\right) \\
H_{\mathrm{R}_{4}} & =\frac{1}{4} l^{e}\left(-1+2 \xi+3 \xi^{2}\right)
\end{aligned}\right.
$$


Author contributions. HRM derived the formulation, implemented it into a code and used it to create and analyze the models in numerical examples $1-3$, performed all the verification case studies, and wrote the article. AK initiated and supervised the work, implemented the formulation into a large-scale computational framework used in numerical example 4 and edited the article.

Competing interests. The authors declare that they have no conflict of interest.

Acknowledgements. Funding from the WoodWisdom ERANET program (PowerBonds project) is gratefully acknowledged. We also thank Anders Eriksson and Elsiddig Elmukashfi for valuable discussions and for comments that greatly improved the manuscript.

Edited by: Amin Barari

Reviewed by: three anonymous referees

\section{References}

Durville, D.: Simulation of the mechanical behaviour of woven fabrics at the scale of fibers, Int. J. Mater. Form., 3, 1241-1251, 2010.

Durville, D.: Contact-friction modeling within elastic beam assemblies: an application to knot tightening, Comput. Mech., 49, 687707, 2012.

Gay Neto, A., Pimenta, P., and Wriggers, P.: Self-contact modeling on beams experiencing loop formation, Comput. Mech., 55, 193 208, 2015.

Konyukhov, A. and Schweizerhof, K.: Geometrically exact covariant approach for contact between curves, Comput. Method. Appl. M., 199, 2510-2531, 2010.

Kulachenko, A. and Uesaka, T.: Direct simulations of fiber network deformation and failure, Mech. Mater., 51, 1-14, https://doi.org/10.1016/j.mechmat.2012.03.010, 2012.

Litewka, P.: The penalty and Lagrange multiplier methods in the frictional 3D beam-to-beam contact problem, Civil and Environmental Engineering Reports, 1, 189-207, 2005.
Litewka, P.: Hermite polynomial smoothing in beam-tobeam frictional contact, Comput. Mech., 40, 815-826, https://doi.org/10.1007/s00466-006-0143-9, 2006.

Litewka, P.: Smooth Frictional Contact between Beams in 3D, in: IUTAM Symposium on Computational Methods in Contact Mechanics, edited by: Wriggers, P. and Nackenhorst, U., IUTAM Bookseries, vol 3, Springer, Dordrecht, 157-176, Springer Netherlands, Dordrecht, https://doi.org/10.1007/978-14020-6405-0_10, 2007.

Litewka, P.: The Beam-to-Beam Contact Smoothing with Bezier's Curves and Hermite's Polynomials, in: Computer Methods in Mechanics, edited by: Kuczma, M. and Wilmanski, K., Advanced Structured Materials, vol 1. Springer, Berlin, Heidelberg, chap. 23, 439-452, https://doi.org/10.1007/978-3-642-052415_23, 2010.

Litewka, P. and Wriggers, P.: Contact between 3D beams with rectangular cross-sections, Int. J. Numer. Meth. Eng., 53, 20192041, https://doi.org/10.1002/nme.371, 2002.

Magnusson, M. and Östlund, S.: Numerical evaluation of interfibre joint strength measurements in terms of three-dimensional resultant forces and moments, Cellulose, 20, 1691-1710, https://doi.org/10.1007/s10570-013-9939-x, 2013.

Meier, C. and Popp, A. andWall, W.: A finite element approach for the line-to-line contact interaction of thin beams with arbitrary orientation, Comput. Method. Appl. M., 308, 377-413, 2016.

Meier, C., Wall, W., and Popp, A.: A unified approach for beamto-beam contact, Comput. Method. Appl. M., 315, 972 - 1010 2017.

Motamedian, H. R.: Beam-to-Beam Contact and Its Application to Micromechanical Simulation of Fiber Networks, PhD dissertation, available at: http://urn.kb.se/resolve?urn=urn:nbn:se:kth: diva-223233 (last access: 19 February 2018), 2018

Wriggers, P. and Zavarise, G.: ON CONTACT BETWEEN THREE-DIMENSIONAL BEAMS UNDERGOING LARGE DEFLECTIONS, Commun. Numer. Meth. En., 13, 429-438, https://doi.org/10.1002/(SICI)10990887(199706)13:6<429::AID-CNM70>3.0.CO;2-X, 1997.

Zavarise, G. and Wriggers, P.: Contact with friction between beams in 3-D space, IInt. J. Numer. Meth. Eng., 49, 977-1006, https://doi.org/10.1002/1097-0207(20001120)49:8<977::AIDNME986>3.0.CO;2-C, 2000. 